\section{REVISTA BRASILEIRA DE QUALIDADE DE VIDA}

\title{
Sexualidade na velhice e qualidade de vida
}

\author{
Sexuality in old age and quality of life
}

\author{
Helena Brandão Viana \\ Faculdade Adventista de Hortolândia- Hortolândia - SP - Brasil \\ hbviana2@gmail.com \\ Vera Aparecida Madruga \\ Faculdade de Educacão Física - UNICAMP - Campinas - SP - Brasil \\ madruga@fef.unicamp.br
}

\begin{abstract}
Resumo
O objetivo deste trabalho, realizado por meio de uma revisão de literatura, foi abordar uma revisão literária sobre a influência da sexualidade na qualidade de vida de idosos, temática pouco discutida nas diferentes áreas de pesquisas sobre envelhecimento. Diante da escassez de bibliografia na cultura brasileira, apresentou-se uma revisão de literatura sobre envelhecimento junto à temática da sexualidade, trazendo uma trajetória histórica sobre estudos de sexualidade ao longo do tempo, discutindo a sexualidade na velhice e apontando como as pesquisas nesta área podem ser úteis para promover o aumento da qualidade de vida do ser humano, em particular, do idoso. As fontes bibliográficas, além de livros clássicos, foram artigos coletados na Base de Dados Ageline priorizando os publicados nos últimos cinco anos, e alguns poucos artigos mais antigos para perfazer uma trajetória histórica da temática. Conclui-se com este estudo que a temática da sexualidade na velhice ainda é pouco estudada no Brasil proporcionando a perpetuação de mitos sobre esse assunto além de informações inadequadas e insuficientes para a população idosa.
\end{abstract}

Palavras-chave: sexualidade, envelhecimento, qualidade de Vida.

\section{Abstract}

The objective of this study, conducted through an extensive literature review, was to review the literature about the influence of sexuality on quality of life, thematic little discussed in the different areas of research on aging. Given the scarcity of literature in Brazilian culture, it was presented a literature review on aging with the theme of sexuality, bringing a historical trajectory about studies on sexuality over time, discussing sexuality in old age and pointing out how the researches in this area can be useful to promote increased quality of life of human beings, particularly the elderly. The bibliographical sources, aside from classic books, were articles collected in the Database Ageline, prioritizing the texts published in the last five years, in addition to a few older articles, to make up a historical background of the theme. We conclude from this study that the theme of sexuality in old age has been barely studied in Brazil, promoting the perpetuation of myths about that subject besides providing inadequate information to the elderly.

Keywords: sexuality, aging, quality of life. 


\section{Introdução}

Sexualidade e afetividade constituem um fenômeno complexo que envolve as esferas biológica, psicológica e social e embora não seja fácil compreender ou medir objetivamente, o amor e o sexo são aspectos fundamentais da vida humana adulta. Segundo Parker (2007) nos últimos 25 anos houve uma real explosão de pesquisas em saúde pública sobre sexualidade. Nunca dificuldades sexuais e temas da sexualidade foram tão priorizados e valorizados pela ciência. Após um breve intervalo por parte da ciência ao assunto sexualidade nos meados do século 20, quando o controverso estudo do Dr. Kinsey foi publicado em 1948 (SCHAIE; BIRREN, 2005), pesquisas focadas em sexualidade diminuíram e quase não houve apoio financeiro para estas (ZEISS, 2010).

Particularmente quando a AIDS se tornou epidêmica nos anos 80, a conseqüência desta longa negligência de pesquisar sobre a sexualidade e a saúde tornou-se aparente, assim como as limitações das estruturas conceituais existentes e as formas dos métodos de pesquisas. Parker (2007) relata que há uma necessidade urgente por formas mais inovadoras para o estudo da vida sexual. Pesquisadores de saúde pública e trabalhadores da área de saúde tem se esforçado em discutir esses assuntos nas últimas décadas, uma nova onda de pesquisa, intervenção e promoção de saúde sexual começaram a tomar forma, usualmente estão crescendo em resposta às necessidades e demandas de comunidades locais que se esforçam em responder construtivamente para uma série de desafios da saúde sexual.

$\mathrm{Na}$ revisão de leituras sobre sexualidade e envelhecimento, visualiza-se a falta de pesquisas nessa área específica. Os estudos existentes sobre sexualidade abordam em poucas páginas a coorte do idoso, e outros sobre envelhecimento, têm voltado seus olhos para a temática da sexualidade em poucos estudos (GOLDSTEIN, 1999; FREITAS et al., 2002; NEGREIROS, 2004; RIBEIRO; JESUS, 2006; FONSECA; RIZZOTTO, 2008; MOURA; LEITE; HILDEBRANT, 2008; SILVA; PEDROSA, 2008).

\section{A Sexualidade no envelhecimento}

Segundo Palmore, Harris e Branch (2005), Hajjar e Kamel (2003) e Kamel e Hajjar (2003) um dos aspectos menos compreendidos da idade matura, tem sido a sexualidade. Como resultado disso, existe crenças e estereótipos equivocados a respeito do comportamento sexual de idosos. Uma das crenças mais comuns é a de que pessoas mais velhas não estão interessadas em sexo e não perdem tempo pensando nisso. A velhice é freqüientemente vista como um período de ausência de sexualidade, no qual, os idosos evitam o envolvimento em atividades sexuais. Reforçando esse ponto de vista, muitos idosos ficam envergonhados ao conversar sobre sua sexualidade por medo de serem mal interpretados em relação à sua moralidade.

A sexualidade é uma parte importante da existência humana, em qualquer etapa da vida. Mas o processo de envelhecimento pode causar algumas mudanças físicas tanto em homens como em mulheres. Estas mudanças algumas vezes afetam a habilidade de ter e usufruir prazerosamente do sexo com outra pessoa.

Embora existam muitas obras sobre envelhecimento, no tocante à temática da sexualidade há pouquíssimas bibliografias e a maioria das pesquisas enfocam questões sobre disfunções e mudanças no funcionamento sexual do homem e da mulher e pouco refletem em como os idosos têm lidado emocionalmente com sua sexualidade (PAPAHARITOU et al., 2008). Mudanças que ocorrem na sexualidade no envelhecimento, incluem fatores psicológicos e de comportamento, que resultam de causas psicológicas ou fisiológicas ou ambas. No entanto, embora o envelhecimento traga mudanças fisiológicas, ele não pode ser visto como a principal causa das mudanças na sexualidade .

Ao realizar pesquisas bibliográficas sobre a sexualidade do idoso foi encontrado quatro obras direcionadas a esta temática no Brasil, três de autores brasileiros: os livros de Maia e Lopes (1995), o de Bacelar (2002) e o de Santos (2003) ; e duas obras traduzidas para o português, a de Butler e Lewis (1985) e Pascual (2002). Em outros livros eventualmente, encontram-se poucas 
páginas sobre a temática, como no tratado de geriatria e gerontologia (FREITAS et al., 2006), publicado mais recentemente, onde há apenas três páginas sobre sexualidade, escrito por Santos (2003), citada anteriormente. Vê-se portanto a necessidade de discutir-se esta temática no Brasil, tão importante para os pesquisadores, a sociedade, e principalmente para a população idosa.

Segundo Umidi et al. (2007) a saúde de pessoas idosas depende de muitos fatores: comorbidades, autonomia funcional, condições sociais e relacionamentos. A esfera emocional-afetiva e a sexualidade desempenham um importante e subestimado papel na saúde delas. Esses pesquisadores realizaram uma pesquisa com 130 idosos. As respostas indicaram que idosos comunicam suas emoções através da esfera afetiva e sexual, com diferentes níveis de desejo por contato físico. As principais variáveis do estudo foram, sexo, estado civil, co-morbidade e uso múltiplo de medicamentos, a percepção do estado de saúde e de si mesmo, experiências passadas, condições culturais e fatores sociais.

O estudo acima teve sete questionamentos e aqui serão apresentados os dois primeiros, que perguntavam aos indivíduos idosos sobre seus desejos por contato físico com um parceiro e sobre o tipo de contato desejado (relação sexual ou apenas ser tocado, abraçado, acariciado). Os indivíduos expressaram desejo de manter as relações físicas e emocional-afetivas, com algumas diferenças. Entre os casados o desejo por contato físico foi maior do que entre os viúvos. Homens desejam mais a relação sexual do que outros tipos de contato físico, enquanto as mulheres desejam a possibilidade da relação sexual quase tanto quanto de outros tipos de contato físico. O estudo também mostrou que os idosos associaram que a sexualidade e afetividade que tiveram ao longo da vida, como um fator que influencia na persistência do desejo atual. Os respondentes também disseram que é preciso dar-se mais atenção às questões ligadas à sexualidade.

Ainda há falta de informação sobre a temática da sexualidade do idoso mesmo por parte dos profissionais de saúde, e isso ocorre mundialmente. Uma pesquisa de Gott, Hinchliff e Galena (2004) realizada na Inglaterra, mostrou que pouco se sabe sobre como os profissionais de saúde percebem e administram problemas sexuais na velhice. Pouco se conhece a respeito das atitudes desses profissionais que são o primeiro ponto de contato para a maioria das pessoas idosas na Inglaterra que vivenciam problemas de saúde sexual. A pesquisa identificou que esses profissionais não conversam pró-ativamente sobre saúde sexual com as pessoas idosas e que, no primeiro atendimento, saúde sexual é tratada da mesma maneira tanto com idosos como com pacientes jovens e não parece ser um assunto considerado adequado para discutir com idosos. A pesquisa apontou também que muitas crenças a respeito de atitudes e comportamento de pessoas mais velhas foram baseadas numa visão estereotipada da velhice e da sexualidade, ao invés de serem baseadas nas experiências pessoais dos pacientes.

No tocante à preocupação do desconhecimento por parte dos profissionais de saúde quanto a esta temática, Johnson (1997), fez um estudo onde questionou aos idosos sobre o que eles poderiam sugerir aos profissionais de saúde no tocante ao atendimento de idosos em dúvidas e questões a respeito da sexualidade. A análise destas respostas resultou num guia para profissionais de saúde. As sugestões foram que os profissionais da área de saúde deveriam gastar tempo quando no atendimento aos idosos, estando disponíveis para discutir o assunto "sexualidade", dando aos idosos total atenção, permitindo que haja tempo para perguntas e mostrando que está à vontade para responder a essas perguntas. Sugeriram ainda que esses profissionais deveriam usar uma linguagem simples, explicando os termos médicos e dando respostas e informações num linguajar mais simples.

Os idosos gostariam de se sentirem confortáveis, podendo expressar emoções e necessidades e não ficando temerosos ou embaraçados ao discutir problemas a respeito da sexualidade idosa. Os profissionais de saúde devem ter a mente aberta e falar abertamente, responder questões diretamente, sem rodeios ou embaraços, mostrando que querem discutir a respeito do assunto, que há interesse por isso e mostrar provas científicas sobre alguns assuntos quando os idosos desejarem. Os profissionais de saúde, segundo os pesquisados, devem estar preparados para ouvir atentamente, mostrando interesse pelos que eles vão falar e enxergar o idoso como indivíduos que têm necessidades sexuais. Deveriam ainda providenciar um ambiente com privacidade; desenvolver 
grupos de suporte e de discussão para os que desejarem com informações precisas; discutir velhos tabus e dar sugestões para possíveis resoluções de problemas.

Os trabalhadores da área de saúde que atendem idosos devem tentar eliminar a idéia que sexo e amor, são só para pessoas jovens, devem mostrar que impulsos sexuais são saudáveis e não desaparecem com o envelhecimento. Tratar os idosos como seres normalmente sexuados e não assexuados e reconhecer que o sexo pode melhorar ainda mais quando envelhecemos, é uma atitude que deveria estar presente no dia a dia dos profissionais da área de saúde que atendem pessoas idosas.

Transmitir às pessoas idosas que procuram ajuda profissional, segurança para discutir a temática da vida sexual e possibilitar-lhes acesso às informações que possibilitem que esse público usufrua plenamente de sua sexualidade é muito importante e pode contribuir para a melhora da qualidade de vida dos mesmos. Muitos nessa fase da vida, ao se separarem ou ficarem viúvos, pensam que não mais devem ter um parceiro ou atividade sexual. Principalmente as mulheres quando na velhice separam-se ou ficam viúvas, deixam de vivenciar a sua sexualidade.

No entanto, muitas mulheres, segundo Anderson (1979, p. 115), encontram no amor maduro, no segundo ou terceiro casamento, satisfação pessoal e sexual às vezes pela primeira vez na vida. Muitas ao envolverem-se em relacionamento sexual na maturidade realizam-se por estarem livres de preconceitos e repressões impostos pela família e sociedade. Elas se encorajam, “[...] querem conhecer melhor a si mesmos, aceitam seus desejos por companhia, amor e sexo como sendo naturais e saudáveis e vão em busca disso".

Um estudo de Nappi e Nijland (2007), envolvendo 1.805 mulheres, que abordou as mudanças na função sexual e comportamento dessas mulheres diante da sexualidade, mostrou que (34\%), ou seja, um terço dessas mulheres experimentaram uma redução da iniciativa na sexualidade, enquanto que, $(53 \%)$ das mulheres relataram que tornaram-se menos interessadas em sexo, mas a maioria da amostra (71\%) afirmou ser importante manter uma vida sexual ativa. Quase metade da amostra do estudo reportou ter contato sexual no mínimo quatro vezes ao mês e relacionou que bem-estar mental e sexual interfere com a auto-estima e prazer de viver.

A auto-imagem, segundo Denney e Quadagno (1992), também influencia na sexualidade na velhice. Quando um idoso sente que não é mais atraente por alguma mudança em seu corpo, isso pode torná-lo menos ativo sexualmente, pois quem não se sente atraente, normalmente toma menos iniciativas em participar de atividades sexuais. Enquanto o idoso não superar a questão, de que mudanças corporais relativas à idade não o tornam uma pessoa não atraente, isso afetará o seu comportamento sexual.

De acordo com Fraiman (1994), muitas mulheres reprimidas sexualmente, com o passar dos anos, na medida em que ganham experiência sexual, mais confiança em si mesmas e no parceiro, podem usufruir maior prazer na relação sexual do que quando eram jovens. Kingsberg (2002), relata que o envelhecimento tem um forte impacto na qualidade do relacionamento e da função sexual. Os impactos psicológicos do envelhecimento após a meia-idade é um assunto importante e deve ser cuidadosamente estudado, pois este conhecimento aumenta a compreensão médica e psicológica da sexualidade de homens e mulheres e pode ajudar no tratamento de algumas disfunções que surgem não só com a idade como também com outras mudanças psicológicos que afetam o ser humano. Segundo o autor é hora de acabar com o estereótipo da mulher de meia-idade para analisar de maneira mais eficiente as questões emocionais e sexuais que aparecem em seus relacionamentos.

\section{A Importância da pesquisa em sexualidade no envelhecimento}

Segundo Zeiss (2010), da Associação Americana de Psicologia, não se discute suficientemente sobre sexualidade no envelhecimento. Sabemos que o envelhecimento traz mudanças importantes e que a sexualidade não é um assunto sobre o qual os idosos tenham muito conhecimento. Entretanto, atualmente se ouve e se vê na mídia propagandas de grandes laboratórios trazendo mudanças na área farmacológica e prometendo melhora na vida sexual dos idosos. De 
repente o mundo foi inundado com informação e evidências que idosos estão vitalmente interessados em sexualidade.

A interação de médicos e psicólogos com pacientes idosos que vão aos centros de saúde em busca de tratamento para mudanças e/ou distúrbios sexuais pode mudar os estereótipos negativos a respeito do envelhecimento nesses profissionais de saúde. Embora muitos idosos percebam que a sexualidade pode ser um importante fator para a qualidade de vida, é mais comum que pessoas mais velhas vivenciem problemas de disfunção erétil do que jovens e muitas vezes relacionam o envelhecimento com perda de virilidade sexual. Por isso o advento de novos fármacos, tem mudado esse quadro. As companhias farmacêuticas também têm investigado uma alternativa medicamentosa para o público feminino.

Segundo Abdo (2004, p. 10), médica psiquiatra, fundadora do Projeto Sexualidade (ProSex) do Instituto de Psiquiatria do Hospital das Clínicas de São Paulo, "a saúde sexual é um dos aspectos mais importantes da vida. Por isso, toda iniciativa para esclarecer um grande número de pessoas acerca da sexualidade merece destaque". Convicta da importância dessa temática, a autora conduziu uma pesquisa com 7.103 participantes de ambos os sexos, com representações das cinco regiões do Brasil, que responderam a 87 questões sobre sexualidade.

Nessa pesquisa acima mencionada, a autora levantou dados relevantes para o estudo da sexualidade no Brasil. Na questão sobre auto-avaliação do desempenho sexual, os resultados mostraram que mulheres idosas se auto-avaliaram melhor do que os homens idosos, comparados às faixas etárias mais jovens, e que as pessoas com mais de 61 anos, não se auto-avaliaram muito pior que os mais jovens. Outro resultado encontrado quanto à sexualidade de pessoas idosas, foi que homens idosos têm mais atividade sexual do que mulheres (o dobro), e também tem mais desejo por atividades sexuais que as mulheres. Ainda de acordo com a pesquisadora, mulheres acima de 61 anos tem mais dificuldade de ficarem excitadas e tem mais dor na relação sexual que mulheres mais jovens, mas são mudanças de um percentual pequeno. Quando comparou homens e mulheres idosos no tocante à prevalência de inibição de desejo sexual, os resultados foram que as mulheres tem uma inibição cinco vezes maior que os homens e que elas tem três vezes mais dificuldade de atingir o orgasmo que eles.

Em outro estudo Ginsberg, Pomerantz e Kramer-Feeley (2005), verificaram que a maioria dos idosos de suas pesquisas mostraram desejo de manter um relacionamento sexual que incluísse tocar, beijar e eles gostariam de ter mais experiências sexuais do que lhes era acessível. Hillman (2000) relata que a atividade sexual na velhice pode estar associada com desejos de receber intimidade emocional, experimentar e usufruir de prazer físico, satisfazer necessidades biológicas, sentir-se jovem, desafiar mitos e estereótipos sociais, restabelecer uma identidade sexual e melhorar sua consciência corporal.

Uma pesquisa da Universidade de Chicago está sendo considerado o primeiro estudo compreensivo sobre a vida sexual de idosos nos Estados Unidos. Essa pesquisa revelou que o estereótipo de que pessoas idosas são assexuadas, não é verdadeiro. Os norte-americanos não desistem das atividades sexuais porque estão envelhecendo. Uma das autoras, Lindau, relata que a discussão sobre sexualidade no envelhecimento tem sido um tabu ainda nos dias atuais. A pesquisa coletou dados de 3.005 adultos, com idades entre 57 a 85 anos, durante uma entrevista de duas horas, nos períodos de Julho de 2005 à Março de 2006, e mostrou que idosos são tão ativos sexualmente quanto são saudáveis. As razões mais comuns para a inatividade sexual foram problemas de saúde do parceiro (LINDAU et al., 2007) .

A pesquisa de Papaharitou et al. (2008), investigou fatores associados com interesse e comportamento sexual em 454 idosos casados, com idades variando de 60 a 90 anos. Mais da metade relatou ainda ter desejo sexual e frequiência de 4 relações sexuais por mês. Gênero, idade, salários, estar ainda apaixonado pelo parceiro foram significantemente associados com interesse e comportamento sexuais. O estudo também apontou que parceiros que se casaram por amor e não por algum tipo de conveniência, tem relações sexuais com mais freqüência. $O$ estudo demonstrou que idosos mantém atividades sexuais e que fatores pessoais, socioeconômicos e interpessoais estão associados com interesse sexual. Pesquisas sobre sexualidade no envelhecimento têm acontecido há 
muitos anos ao redor do mundo, mas no Brasil ainda estamos iniciando esse processo (ABDO, 2004).

Kinsey (1948 apud SCHAIE; BIRREN, 2005), na primeira pesquisa realizada sobre sexualidade no mundo, relatou que para termos evidências sobre comportamento sexual através de uma entrevista é necessária uma sistemática mais direta do que para obtermos evidências sobre hábitos alimentares, por exemplo. O Survey de Kinsey apontou que em homens a freqüência de orgasmos diminui desde a adolescência quase que linearmente em relação à idade.

No entanto, há evidências que alguns homens em seus 90 anos continuam a ter relações sexuais. A diminuição da frequiência sexual em mulheres acontece também pela indisponibilidade do parceiro ou pela ausência deste. Há mais mulheres viúvas do que homens viúvos. Entre aquelas que ainda têm parceiro, existem aquelas cujo parceiro não consegue mais manter relações sexuais ou perdeu o interesse pelo sexo. Por isso a diminuição da frequiência sexual em mulheres mais velhas não pode ser interpretada como uma diminuição do desejo sexual destas e o relatório de Kinsey sobre as mulheres realizado em 1953, apontou a vivacidade da sexualidade de mulheres mais velhas com a presença do ato da masturbação entre as mulheres sem parceiros ou com parceiros inativos. Segundo Schaie e Birren (2005) isto pode ser uma indicação que a sexualidade muda muito devagar e somente em uma pequena extensão em mulheres, durante o processo de envelhecimento.

No Brasil temos a pesquisa de Abdo (2006), realizada com 7.103 pessoas de 18 a 80 anos em 2003, que traçou um perfil sobre a vida sexual dos brasileiros na atualidade. Nesta pesquisa, 96\% dos entrevistados afirmaram que o sexo é importante ou importantíssimo para a harmonia do casal, e uma parcela bem pequena da população feminina $(7,7 \%)$ e masculina $(2,5 \%)$ afirmou não fazer sexo. Os brasileiros em geral tem duas a tres relações sexuais por semana, mas gostariam de ter o dobro e a maioria (63,5\% dos homens, $51,1 \%$ das mulheres) sentem-se à vontade para falar de sexo, contra uma minoria que se diz constrangido ou envergonhado. Das mulheres, $44 \%$ e dos homens $48 \%$, afirmaram que a satisfação proporcionada pelo sexo é intensa. Quanto aos idosos, detectou-se que após os 60 anos de idade, o desejo sexual diminui $20 \%$ nas mulheres e $5 \%$ nos homens, no entanto, mulheres mais velhas tem menos dificuldade de atingir o orgasmo que as jovens. Outro dado importante foi que $43,6 \%$ das brasileiras e $34,9 \%$ dos brasileiros nunca usam preservativo e esse índice diminui para 13,6\% em mulheres e 18,1\% em homens com 61 anos ou mais. Na pesquisa obteve-se a informação que para $80 \%$ ods brasileiros a dificuldade sexual afeta diversos aspectos da vida diária, tais como relacionamento com o parceiro, com os amigos e com os filhos, prejudica a auto-estima, o trabalho e o lazer.

Para Beauvoir (1990) a sexualidade não é somente um conjunto de reflexos que geram emoções e sensações. As atividades sexuais, segundo a autora, têm uma pluralidade de fins. As gratificações que um indivíduo tira de suas atividades sexuais são de grande diversidade e de grande riqueza. Muitas vezes, a pessoa idosa deseja a atividade sexual para remetê-la à juventude e a momentos de extrema felicidade que possuiu em seu passado. Pelo desejo, pode reavivar as cores de seus anos passados e viris. Porém esse desejo só se encontra em idosos que atribuíram ao longo da vida um valor positivo à atividade sexual. Pessoas que repugnavam as atividades sexuais utilizam a desculpa de estar velho para "poder" abandonar a vida sexual ativa. Enfim, para a autora, a vida sexual prolonga-se tanto mais, quanto mais rica e mais feliz tiver sido ao longo do curso de vida.

É comum, mulheres após a menopausa, livres da possibilidade de engravidar, renovarem seu interesse pelos maridos e descreverem que desejam ter, ou algumas já tiveram, uma segunda lua de mel em sua idade madura. Se o casal tem um bom relacionamento afetivo, pode renovar suas atividades sexuais com mais prazer e liberdade diante da não possibilidade de gravidez. E embora alguns estudos tragam que há uma diminuição na atividade sexual após a menopausa, têm-se observado que o desejo sexual nem sempre diminui. (VASCONCELOS et al., 2004; LORENZI; SACILOTO, 2005; GRADIM; SOUZA; LOBO, 2007; TRUDEL, 2008).

Segundo Butler e Lewis (1985), o sexo e a sexualidade são experiências prazerosas, gratificantes e reconfortantes que realçam os anos vindouros, proporcionando um maior bem-estar 
individual. Os autores ainda relatam que o ato sexual é complexo, abrangendo o corpo, a mente e as emoções. Afirmam os autores que mulheres mais velhas, às vezes, se preocupam com a aparência física e temem a perda de seu aspecto atraente e jovem. Algumas pessoas idosas decidem que são feias e indesejáveis e começam a odiar suas aparências. Se o idoso se considera sem atrativos, acabará ficando assim e provavelmente deixará de aproveitar oportunidades, que poderiam trazer encontros sociais e afetivos agradáveis. Para os autores, programas de exercícios, podem melhorar a aparência física e a vida sexual.

Pesquisa realizada por Viana (2003), sobre a percepção da qualidade de vida de pessoas idosas, onde foi utilizado o instrumento da Organização Mundial de Saúde, o WHOQOL - bref, que contém 26 questões, detectou-se que idosos ativos fisicamente se avaliaram mais satisfeitos com sua vida sexual do que idosos inativos fisicamente. Nesta pesquisa acima, outras duas questões apresentaram diferença estatisticamente significante. Uma delas perguntava sobre a aceitação da própria aparência e a outra perguntava sobre quão satisfeito o idoso se sentia consigo mesmo. Nas correlações feitas nesta pesquisa pode-se detectar que os idosos que aceitavam melhor sua aparência física estavam mais satisfeitos consigo mesmo e se avaliaram mais satisfeitos com sua vida sexual. $\mathrm{E}$ os idosos que mais se mostraram satisfeitos com a aparência física, foram os ativos fisicamente, apontando a possível influência da atividade física sobre a imagem corporal, conseqüente satisfação consigo mesmo e com a vida sexual.

\section{Discussão}

$\mathrm{Na}$ realidade, pesquisas recentes têm identificado que enquanto os idosos vivenciam experiências sexuais, eles gostariam de discutir isso com um profissional de saúde, mas a maioria não o faz porque eles se preocupam se isso seria um assunto apropriado para uma pessoa de "sua idade" falar (GOTT; HINCHLIFF; GALENA, 2004). Por outro lado, profissionais de saúde temem iniciar a conversa sobre sexualidade e de alguma forma ofender o idoso. Esses autores acreditam que futuramente esse quadro poderá mudar e os idosos irão querer discutir mais sobre sexualidade com seus médicos e outros profissionais de saúde e isso provavelmente exigirá uma capacitação adicional desses profissionais.

Um estudo feito com médicos na Turquia identificou que os profissionais de saúde têm pouco conhecimento sobre sexualidade no envelhecimento, poucos costumam conversar com seus pacientes idosos sobre a temática da sexualidade e afirmaram que se sentiriam mais confortáveis se o idoso iniciasse a conversa. $\mathrm{O}$ estudo revelou que as mulheres médicas são mais conservadoras do que médicos homens em relação à sexualidade do idoso e têm menos conhecimento que eles (DOGAN et al., 2008).

$\mathrm{Na}$ coleta de dados sobre sexualidade do idoso realizada por Viana (2008), com idosos, cuidadores de idosos e parentes que vivem com idosos, ficou claro a dificuldade que existe em falar sobre essa temática com algumas pessoas. No processo de contato com coordenadores de grupos de terceira idade, muitos se mostraram dispostos e conscientes da importância do tema, porém ao expor aos alunos do que se trataria a pesquisa, alguns idosos afirmaram que isso não é importante e que falar sobre sexualidade é muito embaraçoso. A feição de alguns idosos ao responderem a escala, mostrou a vergonha de falar sobre isso. Porém isso não ocorreu com a maioria dos idosos que foi entrevistada, que se mostrou disposta a falar sobre, e a colaborar com a pesquisa. Foi verificado nesse estudo, que a população idosa brasileira se avalia mais conservadora no tocante à sexualidade no envelhecimento e não conhece muito sobre as mudanças que ocorrem nessa fase, o que de certa forma, acaba interferindo nas tomadas de decisão sobre sua sexualidade.

O envelhecimento corporal traz algumas mudanças. Como regra geral, temos modificações sutis e graduais e a maioria delas não traz problemas para o idoso. Algumas modificações mais dramáticas e súbitas podem indicar problemas sérios de sáude. Pessoas com bons hábitos de saúde e que mantém esses hábitos ao longo da vida, podem experimentar um envelhecimento com qualidade. Um estilo de vida saudável incluindo uma quantidade adequada de atividade física, como alongamentos e caminhadas, bons hábitos alimentares e pouca exposição a fatores estressores 
podem gerar uma velhice com maior bem-estar. Da mesma forma, adultos que tem uma vida sexual ativa, podem manter essa atividade também nos anos da maturidade e velhice com qualidade.

Normalmente, as revisões de literatura sobre a sexualidade idosa tem seguido um padrão, onde discutem sobre os mitos mais comuns existentes, seguidas por informações importantes a serem divulgadas para os idosos e as pessoas com quem eles convivem, profissionais de saúde que trabalham direta ou indiretamente com pessoas mais velhas.

Vasconcelos et al. (2004), relatou a dificuldade de interpretação dos resultados de uma pesquisa sobre sexualidade, onde as pessoas podem dar respostas que julgam ser as certas e não necessariamente a verdade sobre seu comportamento sexual o que por vezes desencoraja pesquisas sobre sexualidade, principalmente junto a população idosa.

Aos olhos de muitas pessoas, idade avançada e intimidade sexual não combinam. Mas a sexualidade pode ser uma parte importante do relacionamento, não importa a idade da pessoa. Revisar a literatura e realizar pesquisas de campo sobre esta temática, a fim de determinar as necessidades dos idosos em relação a sua sexualidade, se faz premente.

Por este motivo é tão importante discutir e pesquisar mais sobre a sexualidade da pessoa idosa no Brasil contribuindo assim, para a melhora da qualidade de vida dessa população, já que a própria Organização Mundial de Saúde elenca a sexualidade como um dos fatores que contribuem para a qualidade de vida (VIANA, 2003).

\section{Referências}

ABDO, C. Descobrimento sexual do Brasil: para curiosos e estudiosos. São Paulo: Summus, 2004.

ANDERSON, B. G. The aging game: success, sanity and sex after 60. Toronto: McGraw-Hill Book Company, 1979.

BACELAR, R. O desejo não tem idade: a sexualidade da mulher idosa. Recife: FASA, 2002.

BEAVOUIR, S. A velhice. 3. ed. Rio de Janeiro: Nova Fronteira, 1990.

BUTLER, R. N.; LEWIS, M. I. Sexo e amor na terceira idade. 2. ed. São Paulo: Summus editorial, 1985.

DENNEY, N. W.; QUADAGNO, D. Human sexuality. 2. ed. Toronto: Mosby Year Book, 1992.

DOGAN, S. et al. Knowledge and attitudes of doctors toward the sexuality of older people in Turkey. International Psychogeriatrics, v.20, p.1019-1027, apr. 2008.

FONSECA, F. B.; RIZZOTTO, M. L. F. Construção de instrumento para avaliação sócio-funcional em idosos. Texto \& Contexto Enfermagem, v.17, n.2, p.365-373, 2008.

FRAIMAN, A. P. Sexo e afeto na terceira idade. São Paulo: Editora Gente, 1994.

FREITAS, M. C. et al. Perspectivas das pesquisas em gerontologia e geriatria: revisão da literatura. Revista Latino-Americana de Enfermagem, v.10, n.2, p.221-228, mar./apr, 2002.

GINSBERG, T. B.; POMERANTZ, S. C.; KRAMER-FEELEY, V. Sexuality in older adults: behaviors and preferences. Age and Ageing, v.34, p.475-480, 2005. 
GOLDSTEIN, L. L. A produção científica brasileira na área da gerontologia: (1975-1999). Revista On-line Biblioteca Prof. Joel Martins, v.1, n.1, 1990.

GOTT, M.; HINCHLIFF, S.; GALENA, E. General practitioner attitudes to discussing sexual health issues with older people. Social Science \& Medicine, v.58, p.2093-2103, 2004.

GRADIM, C. V. C.; SOUSA, A. M. M; LOBO, J. M. A prática sexual e o envelhecimento. Cogitare Enfermagem, v.12, n.2, p. 204-213, abr./jun. 2007.

HAJJAR, R.; KAMEL, H. Sexuality in the Nursing Home, Part 1: Managing abnormal behavior legal and ethical issues. Journal of the American Medical Directors Association, v.4, n.3, p. 152-156, 2003.

HILLMAN, J. L. Clinical perspectives on elderly sexuality. USA: Kluwer Academic, 2000.

JOHNSON, B. Older adults' suggestions for health care providers regarding discussions of sex older adults answer a survey about sexuality and aging. Geriatric Nursing, v.18, n. 2, p. 65-66, 1997.

KAMEL, H.; HAJJAR, R. Sexuality in the Nursing Home, Part 2: Managing abnormal behavior legal and ethical issues. Journal of the American Medical Directors Association, v.4, n.4, p. 203206, 2003.

KINGSBERG. S. A. the impact of aging on sexual function in women and their partners. Archives of sexual behavior. v. 31, n. 5, p. 431-437, 2002.

LINDAU, S. T. et al. A study of sexuality and health among older adults in the United States. The New England Journal of Medicine, v. 357, n.8, p. 762-774, 2007.

LORENZI, D. R. S; SACILOTO, B. Freqüência da atividade sexual em mulheres menopausadas. Revista da Associação Médica Brasileira, v. 52, n. 4, p. 256-260, 2006.

MAIA, M.; LOPES, G. Sexualidade e envelhecimento. São Paulo: Saraiva, 2005.

MOURA, I.; LEITE, M. T.; HILDEBRANT, L. M. Idosos e sua percepção acerca da sexualidade na velhice. Revista Brasileira de Ciências do Envelhecimento Humano, v.5, n.2, p. 132-140, 2008.

NAPPI, R. E.; NIJLAND, E. A. Women's perception of sexuality around the menopause: Outcomes of a European telephone survey. European Journal of Obstetrics \& Gynecology and Reproductive Biology, v.137, n.1, p. 10-16, 2007.

NEGREIROS, T. C. G. M. Sexualidade e gênero no envelhecimento. ALCEU, v.5, n.9, p.77-86, 2004.

PALMORE, E. B.; HARRIS, D.; BRANCH, L. Encyclopedia of ageism. England, Oxford: Routledge, 2005.

PAPAHARITOU, S. et al. Factors associated with sexuality in later life: an exploratory study in a group of Greek married older adults. Archives of Gerontology and Geriatrics, v. 46, n. 2, 191201, 2008. 
PARKER, R. Sexuality, health, and human rights. American Journal of Public Health, v. 97, n.6, p. 972-973, 2007.

PASCUAL, C. P. Sexualidade do idoso vista com novo olhar. São Paulo: Loyola, 2002.

RIBEIRO, L. C. C.; JESUS, M. V. N. Avaliando a incidência dos casos notificados de AIDS em idosos no estado de Minas Gerais no período de 1999 a 2004. Cogitare Enferm, v. 11, n.2, p.113$116,2006$.

SANTOS, S. S. Sexualidade e amor na velhice. Porto Alegre: Sulina, 2003.

SCHAIE, K. W.; BIRREN, J. E. Handbook of the psychology of aging. New York: Academic Press, 2005.

SILVA, A. C. A. P.; PEDROSA, A. S. Sexualidade e Etarismo: análise do discurso em uma lista de debates na Internet. Estudos Interdisciplinares sobre o Envelhecimento, v.13, n.2, p. 221-236, 2008.

TRUDEL, G.et al. The marital life and aging well program: effects of a group preventive intervention on the marital and sexual functioning of retired couples. Sexual Relationship Therapy, v. 23, n.1, p. 5-23, 2008.

UMIDI, S. et al. Affectivity and sexuality in the elderly: often neglected aspects. Archives of Gerontology and Geriatrics, v.44, Suppl. 1, p. 413-417, 2007.

VASCONCELOS, D. et al. A sexualidade no processo de envelhecimento: Novas perspectivas comparação transcultural. Estudos de psicologia, v.9, n.3, p. 413-419, 2004.

VIANA, H. B. A influência da atividade física sobre a avaliação subjetiva da qualidade de vida de pessoas idosas. 2003. 124 f. Dissertação (Mestrado em Educação Física) - Faculdade de Educação Física, Universidade Estadual de Campinas, Campinas.

VIANA, H. B. Adaptação e validação da ASKAS - Aging sexual knowledge and attitudes scale em idosos brasileiros. 2008. 179 f. Tese (Doutorado em Educação Física). Faculdade de Educação Física, Universidade Estadual de Campinas, Campinas.

ZEISS, A. M. Aging and human sexuality resource guide. American Psychological Association. Disponível em <http://www.apa.org/pi/aging/resources/guides/sexuality.aspx $>$.

Acesso em: 25 abr. 2010. 